\title{
PROSPECTIVA DA PÓS-GRADUAÇÃO EM CIÊNCIAS SOCIAIS APLICADAS E HUMANIDADES
}

\author{
Prospective of Graduate Programs on Applied Social Sciences \\ and Humanities
}

\author{
Ivan Rocha Neto \\ Luiza Beth Nunes Alonso
}

\section{Resumo}

São apresentados os resultados e os procedimentos metodológicos do estudo prospectivo desenvolvido pela Capes para servir como base de formulação de políticas e escolhas de investimentos estratégicos. O objetivo foi o de identificar linhas de pesquisa emergentes e avaliar o potencial de expansão do Sistema Nacional de Pós-Graduação (SNPG) para os próximos dez anos. Nos casos específicos das Ciências Sociais Aplicadas e Humanidades foi possível apropriar os resultados de dois estudos prospectivos anteriores com horizonte até 2020: "Projeto do Milênio" e "Brasil em Três Tempos". Este estudo teve base nas fontes de informação da Coordenação de Aperfeiçoamento de Pessoal de Nível Superior (CAPES) (Cursos Recomendados e Cadastro de Discentes) e do Diretório de Pesquisa do Conselho Nacional de Desenvolvimento Científico e Tecnológico (CNPq).

Palavras-chave: Pós-Graduação. Ciências Sociais Aplicadas. Humanidades. Estudo Prospectivo.

\begin{abstract}
This article presents the results and methodological procedures of a study to serve as a basis for policy-making and strategic investments choices. The objective was to identify emerging research issues and assess the potential of expansion of the National System of Graduate Studies in all fields of knowledge, for the next ten years. In the specific case of the Social Applied Sciences and Humanities it was possible to appropriate the results of two recent prospective studies with a horizon to 2020: "Millenium" and "Brazil in Three Moments". The potential of expansion of this system was estimated in accordance with identified emerging research issues, considering information available in the basis of CAPES - Coordination of Improvement of the Higher Education Personnel (Recommended Graduate) and CNPq National Council for Scientific and Technological Development (Directory of Research Groups).
\end{abstract}

Keywords: Graduate Programs. Applied Social Sciences. Humanities. Prospective Study. 


\section{Introdução}

O estudo apresentado neste artigo tem por objetivo identificar linhas de pesquisa emergentes (LPE) e avaliar o potencial de expansão da pósgraduação no Brasil nas Ciências Sociais Aplicadas (CSA) e Ciências Humanas $(\mathrm{CH})$. Para essas áreas foi possível apropriar os resultados de dois estudos prospectivos anteriores ambos com horizontes até 2020: "Projeto do Milênio" e "Brasil em Três Tempos". Este estudo teve base nas fontes de informação da Coordenação de Aperfeiçoamento Pessoal de Nível Superior (CAPES) (Cursos Recomendados e Cadastro de Discentes) e do Conselho Nacional de Desenvolvimento Científico e Tecnológico (CNPq) (Diretório de Grupos de Pesquisa do CNPq - DP). Como em qualquer estudo prospectivo, os resultados são apresentados pela previsão de fatos futuros (neste caso criação de cursos de pós-graduação), acompanhados de suas respectivas probabilidades de ocorrência.

\section{Estudos Prospectivos}

\section{Projeto do Milênio}

Foi desenvolvido e divulgado pela UNU (Universidade das Nações Unidas) e envolveu cerca de 10 mil cientistas, gestores acadêmicos e profissionais dos cinco continentes, orientados às questões de pesquisa para redução da pobreza e fome, bem como à melhoria da qualidade de vida. Portanto de interesse direto das Ciências Sociais Aplicadas e Humanas. Os resultados mostraram que os cientistas de todas as áreas que responderam à consulta DELPHI, metodologia usada no referido estudo, privilegiaram temas de investigação relacionados às tecnologias portadoras de futuro (Nanotecnologia, Biotecnologia e Tecnologia da Informação e Comunicação), desenvolvimento sustentável, além da saúde, produção de medicamentos e alimentos de baixo custo, bem como em relação à superação de dificuldades de comunicação dos cientistas com a sociedade e convivência social. 
A consulta DELPHI é um método clássico de pesquisa de opiniões de pesquisadores, gestores e especialistas sobre questões polêmicas ou com elevado grau de incerteza. Neste caso, o processo de consulta foi iniciado com 14 questões, cujos resultados são destacados neste artigo, segundo duas classes: promessas e riscos. Os resultados foram divulgados em 2002 com horizonte até 2020. Essa metodologia tem sido largamente utilizada desde a década de 1950 (MARTINO, 1975) em estudos de prospectiva tecnológica mediante processo de consultas iterativas a grandes números de especialistas, que permanecem anônimos, mas com realimentação das respostas dos demais participantes para obter convergência e redução de incertezas sobre as questões mais relevantes. Os temas que predominaram estritamente relacionados às Ciências Sociais Aplicadas e Humanidades foram orientados para responder às seguintes questões: Quais os desafios da Ciência Tecnologia e Inovação para melhorar a qualidade de vida e promover o desenvolvimento sustentável? Quais as tecnologias emergentes mais prováveis e com maior impacto econômico e social?

Promessas: Melhoria da inteligência Coletiva; Redução de comportamentos antissociais; Aumento da criatividade; Preservação da diversidade cultural; Redução da Violência; Implicações Econômicas e Sociais das tecnologias (avaliação tecnológica); Pesquisas inter e transdisciplinares; Crenças que desafiam a Ciência; Aumento do controle das empresas no direcionamento das pesquisas; Aumento da importância da educação científica; Melhoria da comunicação dos cientistas com a sociedade em geral; Desenvolvimento de tecnologias educacionais; Universalização do acesso à Internet; Estabelecimento de negócios, respeitando as diferenças culturais; Ética na economia e Redução da corrupção.

Riscos: Disponibilidade generalizada de drogas alucinógenas; Aumento do consumo aos níveis dos EUA; Perda das tradições espiritualistas; Eliminação de todas as formas de segurança de informações; Terrorismo cibernético e Colapso econômico. 
No estudo "Brasil em Três Tempos" (STEINER, 2006), foram desenhados três cenários com horizontes $(2007,2015$ e 2022) que se relacionam ao desenvolvimento sustentável e à educação.

\begin{tabular}{|l|l|l|}
\hline Questões mais relevantes & \multicolumn{2}{|l|}{$\begin{array}{l}\text { Probabilidades } \\
\text { de Ocorrência }\end{array}$} \\
\cline { 2 - 3 } & 2015 & 2022 \\
\hline Qualidade da Educação Básica & 0.32 & 0.41 \\
\hline Universalização da Educação Básica & 0.47 & 0.59 \\
\hline $50 \%$ de redução da criminalidade & 0.3 & 0.4 \\
\hline Crescimento de 2\% ao ano na taxa de empregos & 0.4 & 0.5 \\
\hline Ampliação do Ensino Médio & 0.42 & 0.62 \\
\hline $\begin{array}{l}\text { Redução das desigualdades sociais aos níveis dos países } \\
\text { desenvolvidos }\end{array}$ & 0.41 & 0.46 \\
\hline Inclusão Digital & 0.51 & 0.61 \\
\hline
\end{tabular}

Quadro 1 - Probabilidades de avanços nos cenários 2015 e 2022 das questões relacionadas às Ciências Sociais Aplicadas e Humanidades no Estudo Brasil em Três Tempos.

Fonte: "Brasil Três Tempos" (NAE/CGEE), 2006. Resumido pelos autores.

Alguns dos problemas relacionados às Ciências Sociais Aplicadas e Humanidades já são claros a apontados nos resultados dos referidos estudos. Dentre esses cabe destacar os seguintes: problemas das grandes metrópoles (educação e saúde), além de habitação, saneamento e transportes; continuidade dos desequilíbrios regionais, microrregionais e locais; grandes disparidades de IDH (Índice de Desenvolvimento Humano) e da educação básica IDEB (Índice da Educação Básica); educação para o trabalho; grandes incertezas econômicas; conexão sistêmica entre todos os níveis de educação. 


\section{Metodologia}

A metodologia adotada neste estudo consistiu em dimensionar as potencialidades e as limitações à expansão do Sistema Nacional de PósGraduação (SNPG) nas Ciências Sociais Aplicadas e Humanidades. Portanto, é de natureza quantitativa.

O percurso metodológico envolveu os seguintes passos:

a) Aferição do potencial de ocorrência de novos doutorados para os próximos 10 anos, com base nos mestrados acadêmicos que ainda não evoluíram para este nível, considerando que tem sido rara a criação de doutorados sem a passagem pelo mestrado;

b) Localização das instituições e grupos que desenvolvem atividades nas linhas de pesquisas emergentes, sem ainda oferecer programas de pós-graduação;

c) Identificação dos estágios de evolução das LPE e cruzamento com as áreas de concentração dos programas recomendados pela CAPES e com o Diretório de Grupos de Pesquisa do CNPq;

d) Levantamento das ocorrências de dissertações e teses no Cadastro de Discentes da Capes;

Os critérios para identificação dos estágios de evolução das linhas de pesquisa foram os seguintes:

Emergentes (LPE) - domínio de poucos grupos de pesquisa nacionais, com incidência majoritária de artigos e publicações científicas, ainda com pouca inserção na pós-graduação; neste caso, considerou-se arbitrariamente a ocorrência de até cinco grupos de pesquisa para caracterizar esta condição;

Em Evolução - mais presente nos programas de pós-graduação e com incidência majoritária de artigos e publicações científicas, mas com maior incidência na pós-graduação que nas LP emergentes. Foram consideradas neste caso as ocorrências superiores a seis e inferiores a dez (10) no Diretório de Grupos de Pesquisa do CNPq;

Maduras - já presentes nos cursos de graduação, especialização, mestrado e doutorado, com larga produção de artigos, publicação de livros 
e apropriação econômica e social, isto é, aplicadas na produção de bens e prestação de serviços.

O objetivo é apenas dimensionar as possibilidades reais de criação de novos mestrados e doutorados nos próximos dez anos, por meio do dimensionamento da disponibilidade de grupos de pesquisa para criação de novos programas nas Linhas de Pesquisa Emergentes (LPE). A avaliação da produção de teses e dissertações tem apenas o objetivo de avaliar o grau de inserção na pós-graduação (oferta de cursos, áreas de concentração e produção científica). Esse critério não compreende ou se relaciona com a qualidade da produção acadêmica, nem aos temas que ainda não são pesquisados no País.

Para identificar essas LPE promissoras para criação de cursos de pósgraduação e estimar o potencial de expansão do Sistema Nacional de PósGraduação - SNPG, o estudo foi desenvolvido na seguinte seqüência:

a) Identificação de linhas de pesquisa emergentes;

b) Busca no sítio da Capes (programas recomendados por áreas de concentração) de palavras-chave para iniciar as buscas textuais e literais no Diretório de Grupos de Pesquisa do CNPq (DP);

c) Eliminação para efeito do estudo das linhas de pesquisa genéricas ou maduras ou em evolução, nas quais a pós-graduação já está mais ou menos bem desenvolvida (arbitrariamente mais de seis (6) ocorrências na buscas textuais e literais no DP do CNPq);

d) Cruzamento com os grupos de pesquisa cadastrados no DP do $\mathrm{CNPq}$ usando palavras-chave obtidas pelas áreas de concentração dos programas recomendados pela Capes, com filtro por área;

e) Levantamento do potencial de criação de novos programas de pósgraduação nas LP, pela diferença entre os grupos de pesquisa identificados e o número de programas de pós-graduação com áreas de concentração similares;

f) Relação dos mestrados acadêmicos novos com notas 3, 4 e 5 e que ainda não evoluíram para o doutorado (potencial para o doutorado); 
g) Cruzamento das linhas de pesquisa com ocorrências de dissertações e teses registradas no Cadastro de Discentes no período (2007-2009), no sentido de identificar o grau de inserção na pós-graduação;

h) Cálculo das probabilidades de criação de novos programas de pósgraduação ou áreas de concentração.

$$
\mathrm{P}=\frac{N_{p}-N_{a}}{N_{p}}
$$

$\mathrm{N}_{\mathrm{p}}$ é o número total de grupos de pesquisa identificados no $D P$ e $\mathrm{N}_{a}$ é o número de áreas de concentração identificadas nos cursos recomendados.

De acordo com a teoria das probabilidades, $P=1$ (um) não implica acontecimento certo, nem tampouco $\mathrm{P}=0$ (zero) implica acontecimento impossível.

O Potencial de Expansão - PE (conceito proposto pelos autores) pode ser calculado pela formula: $P E=(N p \times P)$ ou $P E=\left(N_{p}-N_{a}\right)$ que estabelece o limite superior de crescimento de novos mestrados, considerando os atuais grupos de pesquisa. No entanto, é também preciso considerar as dimensões dos grupos e suas superposições em mais de uma área de concentração ou linha de pesquisa. Além disso, há grupos com menos de três pesquisadores, massa crítica mínima necessária para abertura de uma nova área de concentração em um programa já existente. Esses fatores reduzem o potencial calculado dessa forma.

\section{Exemplo: Planejamento Urbano}

Como exemplo da aplicação da metodologia descrita apresenta-se o estudo para a área de Planejamento Urbano. Os resultados das demais áreas são apresentados de forma agregada para limitar o artigo ao espaço permitido pela revista Tecnologia e Sociedade.

O quadro 2 mostra os resultados do cruzamento das ocorrências no Diretório de Grupos de Pesquisa do CNPq (DP) com as áreas de concentração dos programas recomendados pela Capes e o cálculo das 
respectivas probabilidades de criação de novos programas de pósgraduação ou novas áreas de concentração.

\begin{tabular}{|l|ll|l|l|}
\hline LPE & $\begin{array}{l}\text { Diretório } \\
\text { grupos de } \\
\text { Pesquisa } \\
\text { CNPq }\end{array}$ & $\begin{array}{l}\text { Áreas de } \\
\text { do } \\
\text { Concentração e } \\
\text { cursos } \\
\text { recomendados } \\
\text { pela Capes }\end{array}$ & Probabilidade \\
\hline $\begin{array}{l}\text { Cidadania e e } \\
\text { Espaço }\end{array}$ & 2 & 0 & 1 \\
\hline Rede Urbana & 5 & 1 & 0,8 \\
\hline Demografia & 1 & 1 & 0 \\
\hline $\begin{array}{l}\text { Processos } \\
\text { Urbanos }\end{array}$ & 3 & 2 & 0,33 \\
\hline Total & 14 & 5 & 0,64 \\
\hline
\end{tabular}

Quadro 2 - Probabilidades de criação de novos cursos nas linhas de pesquisa emergentes em Planejamento Urbano

Fontes: Diretório de Grupos de Pesquisa do CNPq, 2009, e CAPES, 2009. Elaboração Própria.

\section{Resultados}

LP Maduras ou Genéricas com inserção na pós-graduação: Planejamento Urbano e Regional; Desenvolvimento Sustentável; Cidades; Gestão Urbana; Planejamento Ambiental; Meio Ambiente Urbano; Planejamento Territorial e Espaço Urbano;

Foram identificadas 3 (três) LPE com 9 grupos com potencial para criação de novos programas de pós-graduação: Rede Urbana; Cidadania e Processos Urbanos. Portanto, o potencial de expansão PE $=9$.

Potencial para Doutorado: 9 mestrados acadêmicos com nota 3; e 3 com $(4)=12$ (potencial de criação de 12 doutorados nos próximos dez anos).

Conforme se pode depreender do quadro 2 há pouca densidade de grupos de pesquisa nas linhas emergentes, requerendo esforço de indução por parte da Capes e do CNPq para criação de novos programas de pósgraduação na área, muito importante para responder às questões propostas, sobretudo no estudo "Brasil Três Tempos". 
O quadro 3 mostra as ocorrências de dissertações e teses na área de Planejamento Urbano no sentido de confirmar a inserção das linhas de pesquisa no ambiente da pós-graduação.

\begin{tabular}{|c|c|c|}
\hline Palavras-Chave & \multicolumn{2}{|c|}{$\begin{array}{l}\text { Ocorrências no Cadastro de } \\
\text { Discentes da Capes no } \\
\text { período 2007-2009 }\end{array}$} \\
\hline Rede Urbana & 4 & 1 \\
\hline Processo & 7 & 0 \\
\hline Cidadania & 6 & 0 \\
\hline Espaço & 0 & 0 \\
\hline
\end{tabular}

Quadro 3 - Ocorrências de Dissertações e Teses em Planejamento Urbano no período (2007- 2009) nas LPE

Fontes: Cadastro de Discentes CAPES (2009). Elaboração Própria.

Estes resultados confirmam que estas linhas são realmente emergentes (pequena inserção na pós-graduação) na área de Planejamento Urbano, com poucas ocorrências de dissertações e teses no período 20072009.

\section{Grupos de Pesquisa e Produção Discente nas Linhas de} Pesquisa Identificadas nos Estudos Prospectivos

O quadro 4 mostra as ocorrências de grupos e a correspondente produção discente em relação às questões do "Estudo do Milênio".

\begin{tabular}{|l|l|l|l|l|}
\hline $\begin{array}{l}\text { Palavras- } \\
\text { chave. } \\
\begin{array}{l}\text { Acesso } \\
\text { em: } 12 \\
\text { jun. 2009 }\end{array} \\
\text { de Grupos de Pesquisa } \\
\text { do CNPq }\end{array}$ & $\begin{array}{l}\text { Produção Discente } \\
2007-2009 \\
\text { (Cadastro da Capes) }\end{array}$ \\
\hline \multicolumn{5}{|c|}{ Projeto Millenium } \\
& GP & GP & $\begin{array}{l}(\mathrm{D}+\mathrm{T}) \\
2007- \\
2009\end{array}$ \\
\hline Corrupção & Humanidades & & 0 \\
\hline Violência & 5 & 10 & 429 \\
\hline Pobreza & 111 & 274 & 124 \\
\hline Fome & 69 & 46 & 135 \\
\hline
\end{tabular}




\begin{tabular}{|l|l|l|l|}
\hline Redes Sociais & 63 & 60 & 62 \\
\hline Práticas Antissociais & 61 & 162 & 0 \\
\hline Diversidade Cultural & 9 & 65 & 2 \\
\hline Inteligência Coletiva & 0 & 7 & 0 \\
\hline Criatividade Educação & 2 & 13 & 66 \\
\hline Avaliação Tecnológica & 14 & 6 & 0 \\
\hline Interdisciplinar & 59 & 106 & 165 \\
\hline Transdisciplinar & 2 & 9 & 36 \\
\hline Crença e Ciência & 0 & 1 & 0 \\
\hline Educação Científica & 18 & 81 & 3 \\
\hline Ciência, Tecnologia e Sociedade & 8 & 34 & 0 \\
\hline Ciência, Tecnologia e Democracia & 1 & 0 & 0 \\
\hline Tecnologia Educacional & 8 & 64 & 31 \\
\hline Tecnologia Social & 47 & 38 & 2 \\
\hline Economia e Ética & 3 & 4 & 0 \\
\hline Drogas & 12 & 41 & 35 \\
\hline Desenvolvimento Sustentável & 236 & 108 & 271 \\
\hline Qualidade de Vida. & 71 & 106 & 0 \\
\hline Quadro 4 Gupos de Pesquisa & Produçă & \\
\hline
\end{tabular}

Quadro 4 - Grupos de Pesquisa e Produção Discente em relação às questões do Projeto do Milênio

Fontes: Cadastro de Discentes CAPES, Diretório de Grupos de Pesquisa do CNPq. Elaboração Própria.

O quadro 5 mostra as ocorrências de grupos e a correspondente produção discente em relação às questões do estudo "Brasil em Três Tempos".

\begin{tabular}{|l|l|l|l|}
\hline \multicolumn{4}{|c|}{ Brasil em Três Tempos } \\
\hline & $\begin{array}{l}\text { GP } \\
\text { CSA }\end{array}$ & $\begin{array}{l}\text { GP } \\
\text { Humanidades }\end{array}$ & $\begin{array}{l}\text { D + T } \\
2007- \\
2009\end{array}$ \\
\hline Educação Básica Qualidade & 16 & 42 & 0 \\
\hline Universalização da Educação & 0 & 1 & 0 \\
\hline IDH & 3 & 0 & 5 \\
\hline Criminalidade & 30 & 37 & 57 \\
\hline Inclusão Digital & 20 & 21 & 56 \\
\hline Inclusão Social & 82 & 104 & 95 \\
\hline Urbano & 274 & 208 & 159 \\
\hline
\end{tabular}

Quadro 5 - Grupos de Pesquisa e Produção Discente em relação às questões do Projeto "Brasil em Três Tempos"

Fontes: Cadastro de Discentes CAPES, Diretório de Grupos de

Pesquisa do CNPq. Elaboração Própria. 
No DP do CNPq, as buscas foram feitas com palavras exatas e com restrição de grandes áreas (CSA e $\mathrm{CH}$ ). No Cadastro de Discentes, sem restrição de áreas, menos do caso de drogas, porque as ocorrências poderiam referir-se às áreas de química, farmácia, medicina e outras. Os resultados com baixa inserção na pós-graduação sugerem as seguintes LPE:

CSA: Corrupção; Inteligência Coletiva; Pesquisas e Estudos Transdisciplinares; Tecnologias Educacionais; Avaliação Tecnológica; Qualidade da Educação Básica; Ciência, Tecnologia e Sociedade; Ciência, Tecnologia e Democracia; Crença e Ciência; Universalização da Educação; Drogas e Economia e Ética.

CH: Crenças e Ciência; Universalização da Educação Básica; Estudos de IDH; Inteligência Coletiva, Criatividade e Educação; Avaliação Tecnológica; Estudos Transdisciplinares; Crença e Ciência; Ciência, Tecnologia e Sociedade; Ciência, Tecnologia e Democracia; Economia e Ética.

Os resultados dos quadros 4 e 5 mostram que os temas sobre violência, desenvolvimento sustentável, tecnologia educacional, redes sociais, interdisciplinaridade, transdisciplinaridade, drogas, desenvolvimento sustentável, qualidade da educação básica, criminalidade, inclusão social e digital, não são emergentes, mas continuam atuais, pois apresentam números significativos de grupos de pesquisa e de ocorrências de dissertações e teses.

\section{Resultados (CSA)}

O quadro 6 apresenta os resultados de ocorrências de grupos de pesquisa por área e as probabilidades de criação de novos programas em quaisquer LP nas Ciências Sociais Aplicadas. 
Potencial para Novos Mestrados em CSA para os próximos 10 anos

\begin{tabular}{|l|l|l|l|l|}
\hline $\begin{array}{l}\text { Áreas } \\
\text { Temas }\end{array}$ & $\begin{array}{l}\text { Diretório do } \\
\text { CNPq }\end{array}$ & $\begin{array}{l}\text { Mestrados } \\
\text { CAPES } \\
(2009)\end{array}$ & $\begin{array}{l}\text { Probabilidades } \\
(\mathrm{P})\end{array}$ & $\begin{array}{l}\text { Potencial } \\
\text { P x Np }\end{array}$ \\
\hline Administração & 231 & 45 & 0,8 & 184 \\
\hline $\begin{array}{l}\text { Arquitetura e } \\
\text { Urbanismo }\end{array}$ & 86 & 10 & 0,88 & 75 \\
\hline $\begin{array}{l}\text { Ciência da } \\
\text { Informação }\end{array}$ & 47 & 22 & 0,53 & 24 \\
\hline $\begin{array}{l}\text { Desenho } \\
\text { Industrial }\end{array}$ & 14 & 10 & 0,29 & 4 \\
\hline Direito & 578 & 40 & 0,93 & 537 \\
\hline Comunicação & 477 & 22 & 0,95 & 443 \\
\hline Economia & 467 & 21 & 0,96 & 97 \\
\hline Serviço Social & 115 & 17 & 0,85 & 98 \\
\hline $\begin{array}{l}\text { Planejamento } \\
\text { Urbano }\end{array}$ & 124 & 26 & 0,79 & 149 \\
\hline Turismo & 154 & 5 & 0,97 & 11 \\
\hline Museologia & 13 & 1 & 0,92 & 2080 \\
\hline $\begin{array}{l}\text { Ciências } \\
\text { Sociais } \\
\text { Aplicadas }\end{array}$ & 2306 & 219 & 0,9 & \\
\hline Quadro 6 Potenclal & & & \\
\hline
\end{tabular}

Quadro 6 - Potencial de criação de novos Mestrados nas CSA Fonte: Elaboração Própria com Base no Diretório de Pesquisa do CNPq e CAPES (2008).

O quadro 6 mostra o potencial de criação de novos mestrados, considerando que todos os grupos de pesquisa existentes pudessem criar novas áreas de concentração ou novos cursos.

Exceto nas áreas de Desenho Industrial e Museologia, as probabilidades de criação de novos mestrados estão acima de 50\%.

\section{Potencial para Doutorado nas Ciências Sociais Aplicadas}

O quadro 7 apresenta os resultados agregados em relação ao potencial para novos doutorados nas Ciências Sociais Aplicadas para os próximos dez anos. 


\begin{tabular}{|l|l|l|l|}
\hline Área & $\begin{array}{l}\text { Com nota três } \\
(3)\end{array}$ & $\begin{array}{l}\text { Com notas } \\
\text { quatro (4) e } \\
(5)\end{array}$ & Potencial \\
\hline Administração & 33 & 9 & 42 \\
\hline Arquitetura e Urbanismo & 6 & 3 & 9 \\
\hline Ciência da Informação & 2 & 2 & 4 \\
\hline Desenho Industrial & 9 & 1 & 10 \\
\hline Direito & 27 & 12 & 39 \\
\hline Comunicação & 21 & 2 & 23 \\
\hline Economia & 9 & 8 & 17 \\
\hline Serviço Social & 12 & 5 & 17 \\
\hline Planejamento Urbano & 7 & 3 & 10 \\
\hline Turismo & 3 & 2 & 5 \\
\hline Museologia & 1 & 0 & 1 \\
\hline $\begin{array}{l}\text { Ciências } \\
\text { Aplicadas }\end{array}$ & 130 & 47 & 177 \\
\hline
\end{tabular}

Quadro 7 - Potencial de Doutorado em CSA

Fonte: Elaboração Própria com Base no Diretório de Pesquisa do CNPq e Cursos Recomendados pela CAPES (2009)

O número de cursos de mestrado acadêmico com nota três (3) indica o potencial para novos doutorados para os três próximos triênios, enquanto o total com notas 4 e cinco para as duas próximas avaliações trienais a partir de 2010. Portanto, há potencial de criação de 177 novos doutorados nos próximos 10 anos.

Ocorrências de Dissertações e Teses em Sociais Aplicadas em 2007 e 2008

O quadro 8 mostra as ocorrências de dissertações e teses nas CSA no período 2007-2008.

\begin{tabular}{|l|l|}
\hline Áreas & Ocorrências. \\
& Teses. \\
& Dissertações \\
& Cadastro \\
& Discente \\
$(2007$ & - \\
\hline Administração & $2009)$ \\
\hline Arquitetura e Urbanismo & 3470 \\
\hline
\end{tabular}




\begin{tabular}{|l|l|}
\hline Ciência da Informação & 259 \\
\hline Desenho Industrial & 84 \\
\hline Direito & 2080 \\
\hline Comunicação & 1290 \\
\hline Economia & 1419 \\
\hline Serviço Social & 627 \\
\hline Planejamento Urbano Regional e Demografia & 376 \\
\hline Turismo & 241 \\
\hline Museologia & 18 \\
\hline Quadro - Ocom
\end{tabular}

Quadro 8 - Ocorrências de Dissertações e Teses em Sociais Aplicadas no período (2007-2009)

Fonte: Cadastro de Discentes da CAPES (2009). Elaboração Própria.

Estes resultados mostram que nas áreas das Ciências Sociais Aplicadas identificadas nos estudos prospectivos relevantes para o desenvolvimento sustentável e qualidade de vida, destacam-se as subáreas de Ciências da Informação, Desenho Industrial, Serviço Social, Planejamento Urbano e Museologia que mereceriam esforço de indução por parte da Capes e do CNPq.

Probabilidades de Expansão da Pós-Graduação nas LPE das Ciências Sociais Aplicadas

O quadro 9 mostra o potencial de expansão da pós-graduação em CSA.

\begin{tabular}{|l|l|l|l|l|}
\hline Áreas & $\begin{array}{l}\mathrm{M} \\
(\mathrm{LPE})\end{array}$ & $\mathrm{P}$ & $\begin{array}{l}\mathrm{D}(10) \\
\text { Próximos } \\
10 \text { anos }\end{array}$ & $\begin{array}{l}\mathrm{D}(6) \\
\text { Próximos } \\
6 \text { anos }\end{array}$ \\
\hline Administração & 7 & 0,63 & 33 & 9 \\
\hline $\begin{array}{l}\text { Arquitetura } \\
\text { Urbanismo }\end{array}$ & 15 & 0,56 & 6 & 3 \\
\hline Ciência da Informação & 9 & 0,69 & 2 & 2 \\
\hline Desenho Industrial & 21 & 0,75 & 9 & 1 \\
\hline Direito & 32 & 0,46 & 27 & 12 \\
\hline Comunicação & 18 & 0,62 & 21 & 2 \\
\hline Economia & 32 & 0,44 & 9 & 8 \\
\hline Serviço Social & 14 & 0,52 & 12 & 5 \\
\hline $\begin{array}{l}\text { Planejamento Urbano } \\
\text { Regional } \\
\text { Demografia }\end{array}$ & 13 & 0,54 & 7 & 3 \\
\hline Turismo & & & & \\
\hline
\end{tabular}




\begin{tabular}{|l|l|l|l|l|}
\hline Museologia & 3 & 0,6 & 1 & 0 \\
\hline $\begin{array}{l}\text { Ciências Sociais } \\
\text { Aplicadas }\end{array}$ & 175 & 0,55 & 130 & 47 \\
\hline
\end{tabular}

Quadro 9 - Probabilidades de Expansão das CSA nas LPE e Potencial de Doutorado

Fonte: CAPES, 2008.

Há 0,55 de probabilidade de criação de 175 novos mestrados nas LPE e 177 doutorados em todas as LP, ou seja, a pós-graduação nas Ciências Sociais Aplicadas tem potencial para crescer cerca de $80 \%$ nos próximos 10 anos.

\section{Resultados para $\mathrm{CH}$}

\section{Probabilidades de Novos Programas de Pós-Graduação em}

Humanidades nas LPE para os próximos 10 anos

O quadro 10 mostra o potencial máximo de expansão em $\mathrm{CH}$, considerando que todos os grupos de pesquisa existentes pudessem criar novas áreas de concentração ou novos cursos.

\begin{tabular}{|l|l|l|l|l|}
\hline Áreas & $\begin{array}{l}\text { Diretório do } \\
\text { CNPq }\end{array}$ & $\begin{array}{l}\text { Mestrados } \\
\text { CAPES } \\
(2009)\end{array}$ & Probabilidades & Potencial \\
\hline Educação & 2277 & 52 & 0,98 & 2231 \\
\hline Ciência Política & 69 & 12 & 0,83 & 57 \\
\hline Sociologia & 201 & 13 & 0,93 & 187 \\
\hline Psicologia & 411 & 23 & 0,94 & 386 \\
\hline Humanidades & 2958 & 100 & 0,97 & 2861 \\
\hline
\end{tabular}

Quadro 10 - Potencial de criação de novos Mestrados nas $\mathrm{CH}$

Fontes: Elaboração Própria com Base no Diretório de Pesquisa do CNPq e CAPES (2009).

O quadro 11 mostra as probabilidades de criação de novos mestrados em $\mathrm{CH}$ nas LPE.

\begin{tabular}{|l|l|l|l|}
\hline Educação & 20 & 9 & 0,55 \\
\hline Ciência Política & 48 & 26 & 0,46 \\
\hline Sociologia & 69 & 27 & 0,61 \\
\hline
\end{tabular}




\begin{tabular}{|l|l|l|l|}
\hline Psicologia & 79 & 37 & 0,53 \\
\hline Humanidades & 216 & 99 & 0,54 \\
\hline
\end{tabular}

Quadro 11 - Probabilidades de Novos Mestrados em Humanidades nas LPE

Fontes: Elaboração Própria com Base no Diretório de Pesquisa do CNPq e CAPES, 2008.

Probabilidades de Expansão das LPE em Ciências Humanas e Potencial de Doutorado

O quadro 12 mostra as probabilidades de expansão da pós-graduação em $\mathrm{CH}$ para os próximos 10 anos.

\begin{tabular}{|l|l|l|l|l|}
\hline Áreas & $\mathrm{M}$ & $\mathrm{P}$ & $\begin{array}{l}\mathrm{D}(10) \\
\text { Próximos } \\
10 \text { anos }\end{array}$ & $\begin{array}{l}\mathrm{D}(6) \\
\text { Próximos } \\
6 \text { anos }\end{array}$ \\
\hline Educação & 11 & 0,55 & 34 & 12 \\
\hline Ciência Política & 22 & 0,46 & 10 & 2 \\
\hline Sociologia & 42 & 0,61 & 10 & 1 \\
\hline Psicologia & 42 & 0,53 & 14 & 7 \\
\hline Humanidades & 117 & 0,54 & 68 & 22 \\
\hline
\end{tabular}

Quadro 12 - Probabilidades de Novos Mestrados e Potencial de Doutorado em CH

Fontes: Elaboração Própria. CAPES, 2009.

Probabilidade de $\mathbf{5 4 \%}$ de criação de 117 novos mestrados nas LPE e 90 de doutorados em geral, ou seja, a pós-graduação nas Humanidades tem potencial para crescer cerca de $50 \%$ nos próximos 10 anos.

\section{Considerações Finais}

Este estudo dimensionou o potencial de expansão da pós-graduação nas Ciências Sociais e Humanas (superdimensionado pelas prováveis superposições de grupos em várias LP, ou da ocorrência de outros sem massa crítica mínima para criação de novas áreas de concentração em cursos de pós-graduação), para os próximos 10 anos, bem como as probabilidades de criação de novos mestrados e potencial para doutorado em linhas de pesquisa emergentes, inclusive em conexão com os temas do 
"Estudo do Milênio" e do "Brasil em Três Tempos". Para a maioria dos temas foi mostrado que há significativa inserção na pós-graduação nas linhas de pesquisa emergentes no País.

\section{Agradecimentos}

Os autores agradecem à Capes e à Unesco pelo apoio ao Projeto 914BRA1123, cujos resultados serviram de base para este artigo.

\section{Referências}

CAPES. Projeto 914BRA1123. Atualidade e Tendências do Sistema Nacional de Pós-Graduação. Brasília: Capes, 2009.

CAPES. Coordenação de Aperfeiçoamento de Pessoal de Nível Superior: Cadastro de Discentes. Disponível em: <www.Capes.gov.br> Acesso em: dez. 2009.

CAPES. Cursos Recomendados. Disponível em: <www.Capes.gov.br> Acesso em: dez. 2009.

CNPq. Diretório de Grupos de Pesquisa. Disponível em: <www.cnpq.br> Acesso em: dez. 2009.

MARTINO, J. P. Technological Forecasting for Decisionmaking. New York: Elsevier Policy Sciences Book Series, 1975.

STEINER, J. Estudos Avançados: Knowledge Future Challenges for Brazil.mht. 2006.

UNU. Universidade das Nações Unidas - Millenium. ONU, 2002. 